\title{
Alternative Wege in die Industrie 4.0 - Möglichkeiten und Grenzen
}

\author{
Bernd Kärcher
}

\section{Einleitung}

Jede Aussage zur Industrie 4.0, ihrer Ausgestaltung und ihrer Konsequenzen ist zum heutigen Zeitpunkt notwendigerweise spekulativ. Konkrete Erfahrungen mit Industrie 4.0 im Sinne des anspruchsvollen technologischen Konzepts, das in Wissenschaft, Wirtschaft und Politik diskutiert wird (Promotorengruppe 2013) - gibt es in der Industrie bisher nicht oder nur in Ansätzen.

Es gibt allerdings Erfahrungen aus technologischen und organisatorischen Innovationsprozessen der Vergangenheit, und es gibt Wissen über aktuelle technologische Entwicklungen, insbesondere im deutschen Maschinenbau. Auf diesen Grundlagen soll im Folgenden ein Blick in die Zukunft gewagt werden.

Die Erfahrungen der letzten Jahrzehnte deuten darauf hin, dass es eigentlich immer zwei grundsätzliche Optionen für den Einsatz von Automatisierungstechnik - im weiteren Sinne - gibt: Einen technikzentrierten Weg, der neben der Automatisierung von Arbeitsprozessen selbst sehr stark auf die Überwachung, Kontrolle und ,Steuerung ' der Mitarbeiter durch technische Mittel setzt. Und einen alternativen Weg, bei dem eine ausgewogene Gesamtlösung in den Dimensionen ,Mensch', ,Technik“ und ,Organisation ‘ im Vordergrund steht.

Es wird hier die Position vertreten, dass es hinsichtlich dieser beiden Wege keinen technologischen Determinismus gibt: Es ist nicht so, dass ,die technische Entwicklung die Unternehmen dazu zwingen würde, den einen oder anderen dieser Wege zu gehen. Unternehmen können entscheiden, welchen Weg sie gehen wollen. Sie können auch entscheiden,

Der Originaltext dieses Beitrags wurde überarbeitet. Das vollständige Korrekturverzeichnis finden Sie am Ende des Buchs und online unter http://dx.doi.org/10.1007/978-3-662-45915-7_16.

B. Kärcher $(\bowtie)$

Festo AG \& Co. KG, Abteilung CR-MC, Leitung Research Mechatronic Components,

Ruiter Strasse 82, 73734 Esslingen, Deutschland

e-mail:Kch@de.festo.com

(C) The Author(s) 2015

A. Botthof, E.A. Hartmann (Hrsg.), Zukunft der Arbeit in Industrie 4.0,

DOI 10.1007/978-3-662-45915-7_5 
nach welchen Kriterien sie entscheiden wollen: Betriebswirtschaftliche Kriterien - kurz-, mittel- oder langfristig orientiert - oder auch ethische und soziale Kriterien.

Die Automatisierungslösungen von Festo sind grundsätzlich für beide Wege in die Industrie 4.0 geeignet.

Ich denke aber auch, dass für Deutschland mit seiner hochentwickelten Industrie und seinen hochqualifizierten Beschäftigten - egal ob Hochschulabsolventen oder beruflich Qualifizierte - der zweite, ganzheitliche Weg, den auch Festo geht, besonders wichtig ist. Deshalb möchte ich auf technische Lösungen für dieses Szenario einen besonderen Akzent legen und dies durch Beispiele aus einem aktuellen Forschungsprojekt illustrieren.

Festo steht nicht nur für Automatisierungstechnik, sondern ebenso - für viele Menschen sogar noch mehr - für Didaktik. Deswegen, und weil Festo als Anwendungsbetrieb moderner Technik auch selbst betroffen ist, liegt es für mich nahe, auch einige Vermutungen - , educated guesses ‘ - zu zukünftigen Qualifikations- und Qualifizierungsbedarfen im Kontext der Industrie $4.0 \mathrm{zu}$ äußern.

Zum Abschluss dieses Beitrags möchte ich einige Anregungen für mögliche Handlungsweisen geben, die den Weg in die Industrie 4.0 für Unternehmen und Beschäftigte besser begehbar machen könnten.

\section{Zwei Wege in die Industrie 4.0}

In den Achtziger- und Neunzigerjahre des zwanzigsten Jahrhunderts wurde eine intensive Debatte um die rechnerintegrierte Fertigung - CIM - geführt. Auch damals standen sich zwei alternative Szenarien gegenüber.

Das eine Szenario orientierte sich an der Vision einer wenn auch nicht menschenleeren, so aber doch weitgehend automatisierten Fabrik. Komplexe IKT-Technik - als zentrale Ressource ausgelegt - sollte das Rückgrat bilden für eine durchgehende Daten- und Automatisierungskette von der Konstruktion (CAD, Computer Aided Design) über die Produktionsplanung (CAP, Computer Aided Planning) bis zur Produktion (CAM, Computer Aided Manufacturing). Neben der unmittelbaren Automatisierung von Arbeitsprozessen sollten technische Systeme in diesem Szenario auch zur Kontrolle und Verhaltenssteuerung der - noch verbliebenen - Mitarbeiter dienen.

Ein alternatives Szenario - auch, humanzentriertes CIM (H-CIM, z. B. Bey et al. 1995) genannt - betonte demgegenüber den Aspekt, dass Menschen weiterhin eine zentrale Rolle im Produktionsprozess spielen werden - und auch spielen sollen. Konzepte wie Gruppenarbeit, Werkstattprogrammierung von Werkzeugmaschinen (z. B. Blum und Hartmann 1988) und anderen Produktionsanlagen sowie die Beteiligung der Mitarbeiter (z. B. Sell und Fuchs-Frohnhofen 1993) an der Gestaltung und Einführung von Automatisierungslösungen spielten in diesem Kontext eine Rolle.

Die technologischen Grundlagen haben sich seitdem verändert. Um nur einen zentralen Aspekt zu benennen: Die damaligen CIM-Konzepte gingen von einer sehr zentralistischen, ,top-down ‘ orientierten IT-Struktur aus. Ganz im Gegensatz dazu setzen heutige Entwicklungen - unter den Stichworten autonome Systeme und cyber-physikalische Systeme - 
auf dezentrale Automatisierung. Oder in Bildern gesprochen: der ,Kommandobrücke ‘ der früheren CIM-Philosophie steht nun ein ,Marktplatz' gegenüber, auf dem autonome technische Systeme dezentral und, vor Ort' Lösungen für Produktionsprobleme, aushandeln ".

Dennoch wird heute ganz ähnlicher Weise, wie oben am Beispiel ,CIM‘ dargestellt, über Szenarien für die Industrie 4.0 diskutiert. Und das ist auch richtig so, denn die grundsätzlichen Gestaltungsfragen stellen sich heute genauso:

- Sollen die technischen Systeme den Menschen ersetzen - beziehungsweise gängeln oder sollen sie ihn unterstützen?

- Möchten wir den Menschen als ,Bediener' der Technik sehen, oder als ,Nutzer“?

- Soll die Flexibilität, die Unternehmen in ihren Produktionsabläufen brauchen, durch flexible Technik realisiert werden, durch flexible Menschen, oder durch eine sinnvolle Kombination aus beidem?

Die technischen Systeme die heute - auch bei Festo - als Elemente der Industrie 4.0 entwickelt werden, können in beiden Szenarien - dem technikzentrierten wie dem ganzheitlichen - eingesetzt werden.

Es ist auch möglich, nach beiden Szenarien in Deutschland wettbewerbsfähig zu produzieren; dies gilt - in gewissen Grenzen - auch über unterschiedliche Branchen und Produkte hinweg.

Die Entscheidung für den einen oder den anderen Weg treffen Unternehmer und Unternehmen nach ihren jeweils eigenen Kriterien. Dabei spielen Philosophien der - engeren oder erweiterten - Wirtschaftlichkeitsbetrachtung ebenso eine Rolle wie ganz grundlegende Werte der Unternehmen.

\section{Merkmale einer humanzentrierten Gestaltung der Mensch-Technik-Interaktion}

\section{Grundlegende Gestaltungsfragen}

Grundlegende Gestaltungsfragen für die Zukunft der Arbeit wurden oben schon kurz und plakativ formuliert.

Der Mensch bleibt ein integraler und unverzichtbarer Bestandteil der Produktionswelt der Zukunft, denn er ist der flexibelste und intelligenteste Teil der heutigen und auch der künftigen Fabrik. Mit der Industrie 4.0 wandern Mensch und Technik noch enger zusammen. Festo forscht deshalb an Lösungen, bei denen der Mensch unmittelbar mit der Technik interagieren kann.

Dabei wird sich die Arbeitswelt natürlich verändern. Einige der heutigen Tätigkeitsfelder wird es in der Zukunft nicht mehr geben, aber dafür werden neue Tätigkeitsfelder dazukommen. Der Mitarbeiter wird abwechslungsreichere und interessantere Tätigkeiten ausüben. Möglicherweise werden manche Tätigkeiten auch schwieriger, was heute noch 
nicht abzuschätzen ist. Insgesamt ist Anpassung gefragt. Der Mitarbeiter muss nicht unbedingt mehr Qualifikationen aufweisen können, sondern vor allem andere als heute.

Diese Aspekte sollen nun noch etwas systematischer betrachtet werden. ${ }^{1}$

Eine ganz grundsätzliche Frage betrifft die Organisationsphilosophie des Unternehmens. Soll sie eher zentral oder dezentral sein? Sollen Entscheidungen eher auf höheren Ebenen der Organisation fallen oder auch vor Ort, zum Beispiel in der Produktion oder im Service? Soll die Transparenz über alle Vorgänge - auch im Detail - bis in die höchsten Entscheidungsebenen hergestellt werden, oder kann die Organisation damit leben, dass dezentrale Handlungen und Entscheidungen als ,black box ' betrachtet werden können?

Zweitens spielt das ,Bild der Arbeit', des arbeitenden Menschen in den Köpfen der Entscheidungsträger eine wichtige Rolle. Werden die Mitarbeiter als kompetent Handelnde und (Mit-) Entscheidende gesehen, oder als Produktionsressourcen, die möglichst gut gesteuert und kontrolliert werden müssen? Damit zusammenhängend: Sind Menschen Nutzer der Technik oder ihre Bediener?

Dieses mentale Bild der Arbeit hängt natürlich ganz entscheidend davon ab, welche Arten von Beschäftigten, mit welchen Qualifikationsniveaus vorhanden, verfügbar oder auch gewünscht sind. Darauf wird weiter unten zurückzukommen sein.

Die Organisationsphilosophie und das Bild der Arbeit hängen natürlich miteinander zusammen. Und beides hat Auswirkungen auf die Gestaltung der Technik.

Auch bei der Technikgestaltung sind - cum grano salis - zwei paradigmatische Wege zu erkennen, die sich plakativ an der Beantwortung folgender Fragen festmachen lassen: Sollen vornehmlich Informationen über die Menschen erzeugt, verarbeitet und aufbereitet werden oder für die Menschen? Sollen menschliche Fähigkeiten ersetzt oder unterstützt beziehungsweise verstärkt werden?

Im Rahmen einer zentralistischen Organisationsphilosophie wären die jeweils ersten, in einer dezentralen Philosophie die jeweils zweiten Antworten naheliegend.

Manchmal wird auch von technikzentrierten gegenüber menschzentrierte Gestaltungsphilosophie gesprochen. Wenn auch Parallelen zu erkennen sind zu den Unterscheidungen, die ich oben getroffen habe, möchte ich diese Begrifflichkeit nicht übernehmen, weil Technik für beide Wege der Gestaltung der Arbeit in der Industrie 4.0 wichtig sein wird. Auch der Mensch wird in beiden Fällen wichtig sein, allerdings in unterschiedlichen Rollen, wie oben beschrieben.

Diese eher theoretischen Betrachtungen sollen im Folgenden illustriert werden anhand eines konkreten und aktuellen Projektbeispiels. ${ }^{2}$ Es wird schnell offenkundig werden, dass sich dieses Beispiel an dem zweiten Weg orientiert: Eine eher dezentrale Organisation mit kompetenten Mitarbeitern, deren Handlungsfähigkeit durch neue technische Systeme unterstützt werden soll.

\footnotetext{
${ }^{1}$ Vgl. zum Folgenden auch den Beitrag von Hartmut Hirsch-Kreinsen „Entwicklungsperspektiven von Produktionsarbeit" in diesem Band.

${ }^{2} \mathrm{http} / / / \mathrm{www}$. esima-projekt.de/.
} 


\section{Projektbeispiel ESIMA}

Das Projektakronym ESIMA steht für „Optimierte Ressourceneffizienz in der Produktion durch energieautarke Sensorik und Interaktion mit mobilen Anwendern“. Es handelt sich dabei um ein Verbundprojekt, das im Rahmen des Forschungsprogramms IKT 2020 im Gebiet „Energieautarke Mobilität - Zuverlässige energieautarke Systeme für den mobilen Menschen“ vom Bundesministerium für Bildung und Forschung gefördert wird. Das Projekt läuft seit dem 01.07.2013 und wird plangemäßzum 30.06.2016 enden.

In diesem Projekt kooperiert Festo mit anderen Firmen (Varta, C4C Engineering $\mathrm{GmbH}$, Daimler, EnOcean) sowie Forschungseinrichtungen (Institut für Mikro- und Informationstechnik der Hahn-Schickard-Gesellschaft e.V., Helmut Schmidt Universität Hamburg, Technische Universität Braunschweig).

Im Projekt ESIMA werden Hardware- und Softwaremodule entwickelt, mit denen die Interaktion von Mensch und Maschine soweit vereinfacht werden soll, dass die Nutzer zu jedem Zeitpunkt über Maschinenzustände und Verbräuche von Ressourcen informiert sind. Mit den vorliegenden Daten sind Optimierungen an Produktionsanlagen einfacher durchführbar. Zum Beispiel können Fehler schnell erkannt und behoben werden. Der Verbrauch an Energie und Materialressourcen lässt sich einfacher und transparenter nachverfolgen, sodass auch in diesem Bereich Optimierungen vereinfacht realisiert werden können.

Ein wichtiger Bestandteil des Projekts ist die Entwicklung energieautarker Funksensoren, deren Anbringung in Produktionsanlagen möglichst einfach und ohne Veränderung der Anlagenstruktur realisierbar sein soll. Parallel werden Softwaremodule entwickelt, die einen rollenbasierten Zugang zu den generierten Informationen erlauben. Je nach Anforderung kann somit eine zielgerichtete Interaktion zwischen Anlage und Nutzer erfolgen.

Mit Hilfe dieser drahtlosen Sensoren wird der Energieverbrauch von Maschinen einfacher zu erfassen sein. Die ermittelten Werte werden auf einem mobilen Endgerät im Produktionsumfeld dargestellt. Die Mensch-Technik-Kommunikation soll also über ein dezentrales Informationssystem erfolgen. Zur Visualisierung der Energiekennwerte und Verbrauchstrends werden mobile Geräte wie Tablet PCs verwendet. Dadurch kann der Werker an der Maschine direkt den Energieverbrauch beurteilen und gegebenenfalls aktiv werden. Diese Informationen waren bisher nur für zentrale Abteilungen beziehungsweise höhere Hierarchieebenen verfügbar.

Abbildung 1 zeigt das Grundkonzept von ESIMA: Daten werden erfasst, analysier und verarbeitet, Kennwerte werden gebildet und visualisiert, als Grundlage für die Optimierung der Prozesse durch die Arbeitenden vor Ort.

Diese Grundphilosophie gilt nicht nur für dieses Projekt und den speziellen Fall der Optimierung im Hinblick auf Energieeffizienz. Für Festo ist dies vielmehr eine ganz grundsätzliche Herangehensweise an die Fabrikautomatisierung, die folgenden Prinzipien folgt:

- Gestaltung und Optimierung der Produktionsprozesse möglichst dezentral vor Ort

- Menschliche Kompetenz als zentrale Ressource für die Produktionssteuerung und -optimierung 


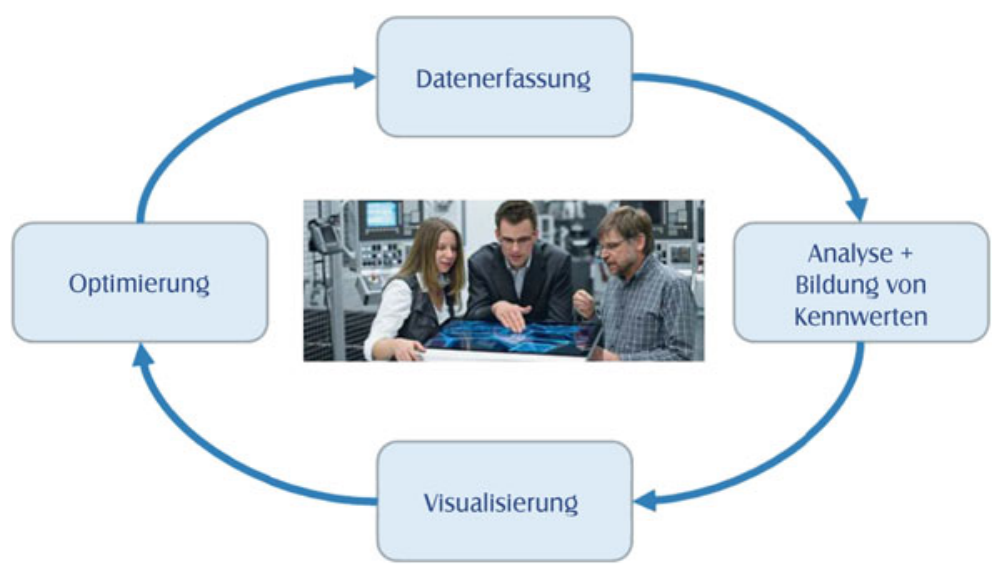

Abb. 1 Grundprinzip des ESIMA-Konzepts, Quelle: Festo AG \& Co. KG

- Technische Systeme als ,Kompetenzverstärker “ für die Nutzer, in zweifacher Hinsicht: Die schon vorhandene Kompetenz der Nutzer wird ernst genommen und in den Mittelpunkt des Konzepts gestellt. Zugleich bieten die Analyse- und Visualisierungsinstrumente Gelegenheit zum kontinuierlichen Weiterlernen in der Arbeit.

\section{Konsequenzen für Qualifikations- und Qualifizierungsbedarfe}

Ganz am Anfang dieses Beitrags wurde darauf hingewiesen, dass heute noch niemand genau sagen kann, wie die Industrie 4.0 aussehen wird, einfach weil noch niemand Erfahrungen damit hat. Dies gilt ebenso für die Qualifikationsbedarfe, die mit Industrie 4.0 möglicherweise einhergehen werden.

Auch die zweite Einschätzung, die dort geäußert, gilt hier analog: Es gibt kein ,Naturgesetz', nach dem sich die zukünftige Realität vorherbestimmen lässt. Die Zukunft wird von vielen Entscheidungen abhängen, die in Politik, Wissenschaft und insbesondere in der Wirtschaft getroffen werden.

Aber ebenso wie für Arbeitsorganisation und Technikgestaltung versucht wurde, anhand einiger Überlegungen und eines Beispiels einen Blick in die Zukunft zu werfen, soll auch eine vorsichtige Spekulation über die Rahmenbedingungen zukünftiger Qualifikationsbedarfe in der Industrie 4.0 gewagt werden.

Ich möchte mich dabei stützen auf Ergebnisse eines internationalen Workshops zum Thema ,Using Technology Foresights for Identifying Future Skills Needs', den die Internationale Arbeitsorganisation (International Labour Organization, ILO) im Sommer 2013 gemeinsam mit der Moscow School of Management SKOLKOVO in Moskau veranstaltete. 


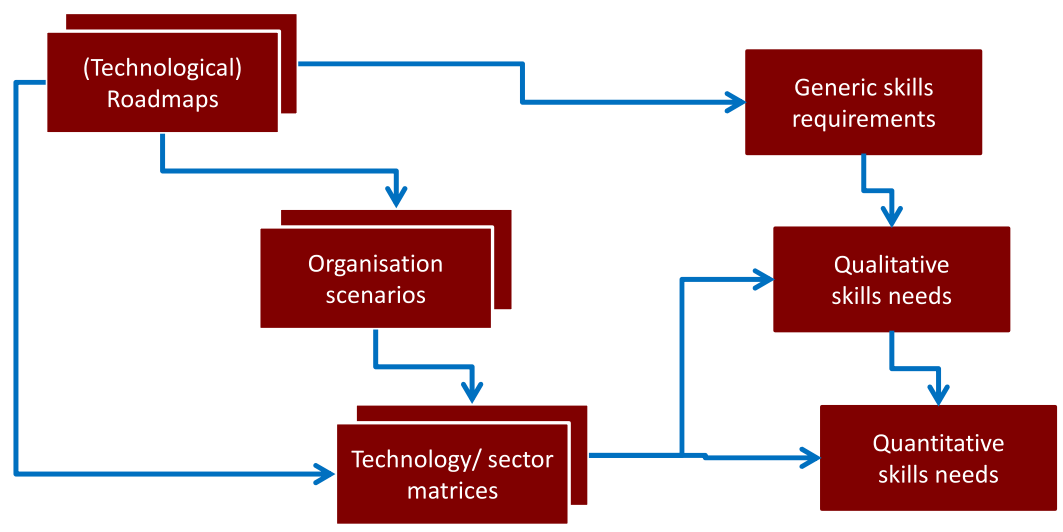

Abb. 2 Methodik zur Qualifikationsbedarfsanalyse basierend auf Technologieroadmaps, Quelle: Hartmann und Bovenschulte (2013), S. 29

Einer der Beiträge hat explizit Qualifikationsbedarfe für Industrie 4.0 zum Gegenstand und beinhaltet auch einen methodischen Vorschlag zur Ermittlung dieser Bedarfe (Hartmann und Bovenschulte 2013; alle folgenden Ausführungen in diesem Abschnitt stützen sich auf diesen Beitrag).

Abbildung 2 zeigt einen Überblick über die dort vorgeschlagene Methodik. Ausgangspunkt sind zunächst Technologieroadmaps. Auch wenn Technologien nicht deterministisch wirken, können sicherlich einige Aussagen über ,Leitplanken` der Entwicklung getroffen werden.

Die Autoren nutzen Materialien der European Technology Platform on Smart Systems Integration (EPoSS) ${ }^{3}$ der European Technology Platform für Robotik (EUROP) ${ }^{4}$ und der International Electrotechnical Commission (IEC) ${ }^{5}$ als Grundlage einer technologischen Vorausschau.

Es werden in Anlehnung an die Technologieroadmap von EPoSS drei ,Generationen smarter Systeme dargestellt. Vereinfacht gesagt, entspricht demnach die erste Generation smarter Systeme der höchstentwickelten Automations-, Steuerung- und Regelungstechnologie, wie wir sie heute vorfinden. Die zweite Generation smarter Systeme wird u. A. über wesentlich erweiterte Funktionen des maschinellen Lernens verfügen. Systeme der dritten Generation schließlich werden charakterisiert sein durch Wahrnehmungs-, Denk- und Handlungsleistungen, die sich immer weiter der menschlichen Leistungsfähigkeit annähern werden.

Gerade im Hinblick auf die dritte Generation smarter Systeme wird für den Teilbereich ,Robotics \& Factory Automation“ eine besonders dynamische Entwicklung angenommen.

\footnotetext{
${ }^{3}$ http://www.smart-systems-integration.org.

${ }^{4}$ http://www.robotics-platform.eu.

${ }^{5}$ http://www.iec.ch/.
} 
Abb. 3 Der bionische Handling-Assistent von Festo als ein Beispiel bionischer und inhärent sicherer

Automatisierung, Quelle: Festo AG \& Co. KG

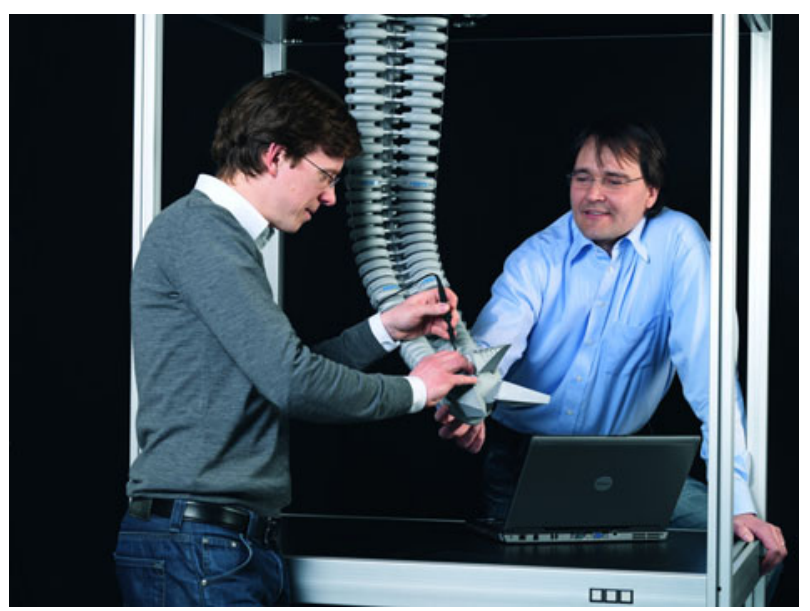

Auch die Robotik-Technologieplattform EUROP hat eine Technologieroadmap vorgelegt. Für die Industrie 4.0 sind dabei besonders die Funktionsbereiche ,Cooperating Robots \& Ambient Intelligence“ und ,Planning ‘ von Interesse. Eigenschaften künftiger robotischer Systeme werden in diesen Funktionsbereichen u. A.: wie folgt beschrieben:

- Distributed control

- Inter-agent communication

- Application of swarm theories/swarm intelligence

- Skill based/learning based automation

- Autonomous planning for tasks of high dimensionality

- Interactive learning from human partners

Die Autoren identifizieren die Robotik als ein besonders relevantes und dynamisches Technologiefeld und benennen als wichtige Aspekte kooperierende Roboter und Fragen der , weichen Automatisierung' (z. B. inhärente Sicherheit durch weiche und flexible Aktuatoren - etwa ,Elefantenrüssel' - robotischer Systeme).

Auch für Festo als Entwickler von Automatisierungslösungen sind diese Fragen von zentraler Bedeutung. Der ,Elefantenrüssel ' als ein Element, weicher' Automatisierung findet seine konkrete Umsetzung im bionischen Handling-Assistenten von Festo ${ }^{6}$ (Abb. 3).

Auch in anderer Hinsicht könnte die Bionik in Zukunft eine wichtigere Rolle spielen für die Entwicklung von robotischen Systemen mit ,nahezu menschlichen“ Fähigkeiten der Wahrnehmung, Kognition und Motorik.

Aufbauend auf diesen Trendprognosen formulieren die Autoren Hypothesen hinsichtlich künftiger qualitativer (im Unterschied zu quantitativen) Qualifikationsbedarfe.

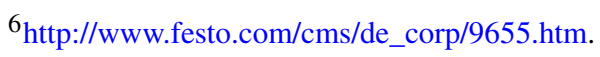


Zunächst wird das Thema ,Sicherheit‘ als Querschnittsqualifikation für viele Berufe und Hochschulqualifikationen im Produktionsbereich genannt. Man denke hier nur an die flexible Arbeitsteilung zwischen Mensch und kooperierendem Roboter: Wenn es keine festgelegten Arbeitsabläufe gibt, muss jede Situation im Arbeitsprozess selbst neu unter Sicherheitsaspekten beurteilt werden. Schutzzäune zwischen Menschen und Robotern wird es dann auch nicht mehr geben, es sind also neue Sicherheitskonzepte gefragt.

Weiterhin könne ein neuer dualer Ausbildungsberuf - im Hinblick auf die zunehmende Verschmelzung von Mechanik, Elektronik und Informatik in der Industrie 4.0 - der ,Industrieinformatiker" sein. Zu klären sei hier die Beziehung zum bestehenden Berufsbild des Mechatronikers. Eine weitere Frage sei, ob - alternativ oder zusätzlich zum Ausbildungsberuf - ein Weiterbildungsberuf geschaffen werden könnte, auch als Weiterbildungsoption für Mechatroniker.

Als ein mögliches zukünftiges hochschulisches Bildungsangebot wird ,Industrielle Kognitionswissenschaft" vorgeschlagen, etwa als Masterprogramm. Zentrale Inhalte könnten hier verteilte Sensor-/Aktornetze, Robotik, Wahrnehmung (z. B. 3D-Sehen) und Kognition (z. B. Handlungsplanung, Kooperation, Schwarmintelligenz) sein.

In ähnlicher Weise sei eine Spezialisierung in ,Automationsbionik' denkbar, die sich ebenfalls auf Robotik beziehe mit Akzenten in der Aktorik (z. B. künstliche Muskeln, Gliedmaßen und Organe) und ebenfalls Aspekten der Wahrnehmung und Kognition aus einer eher biologischen Perspektive.

Der nächste Prozessschritt in Abb. 2 betrifft Organisationsszenarien. Hier diskutieren die Autoren genau die Fragen, die auch in diesem Beitrag weiter oben ausführlich angesprochen wurden: Zentrale versus dezentrale Organisation, Überwachung versus Ermächtigung des Menschen. Und auch die zentrale Aussage dieses Beitrags findet sich dort wieder: Technologische Entwicklungen wirken nicht deterministisch, wie ein ,Naturgesetz auf die Qualifikationsentwicklung. Diese Wirkung wird vielmehr durch die Organisationsszenarien entscheidend moduliert.

Um schließlich quantitative Aussagen zu Qualifikationsbedarfen treffen zu können, müssen unterschiedliche Branchen berücksichtigt werden. Die Veränderungen, die mit Industrie 4.0 einhergehen, werden sich sehr unterscheiden zwischen Unternehmen, die Automatisierungstechnologie entwickeln und herstellen, denen, die sie vornehmlich anwenden, und schließlich denen, die - wie Festo - sowohl als Hersteller wie als Anwender stark betroffen sein werden.

Zur Entwicklung solcher quantitativer Prognosen schlagen die Autoren TechnologieBranchen-Matrizen vor, die die oben umrissenen Abhängigkeiten abbilden. Sie müssen aber einräumen, das dies noch Zukunftsmusik ist: Eine solche Methodik wurde bisher noch nicht entwickelt. Hier besteht also noch erheblicher Forschungs- und Entwicklungsbedarf. Damit ist übergeleitet zum letzten Abschnitt, in dem neben einem Fazit auch ein Ausblick gegeben werden soll. 


\section{Fazit und Ausblick}

Technik - speziell: Automatisierungstechnik - , an sich“ ist weder ,gut' noch ,böse‘. Welche Auswirkungen die technologischen Entwicklungen haben werden, hängt im Wesentlichen von Entscheidungen der Unternehmen ab. Diese Entscheidungen beziehen sich immer auch auf die Wahl zwischen zwei Wegen oder Szenarien. Im ersten Szenario bekommen die Mitarbeiter vor Ort Informationen und Kompetenzen. Im zweiten Szenario werden die Mitarbeiter immer perfekter in Prozessen überwacht und benötigen weder Kompetenzen noch Fähigkeiten. Beide Wege sind möglich, auf beiden Wegen kann in Deutschland wettbewerbsfähig produziert werden.

Festo hat allerdings - orientiert an seiner Unternehmensphilosophie und seinen Tätigkeitsfeldern - eine eigene Sicht auf die Industrie 4.0.

Nach dieser Sichtweise wachsen mit der Industrie 4.0 Mensch und Technik noch enger zusammen. Die Robotik der Zukunft interagiert mit dem Menschen und weicht ihm durch intelligente Sensorik aus. Durch wachsende Intelligenz stellen diese Maschinen eine immer geringere Gefahr im Umgang mit dem Menschen dar und unterstützen ihn darüber hinaus durch Entlastungen bei seiner täglichen Arbeit, zum Beispiel in der Montage. Der preisgekrönte Bionische Handling-Assistent oder die ExoHand, beide von Festo, sind heute schon Vorreiter dieser Entwicklung.

Die Technik wird intelligenter und adaptiver und ist zunehmend in der Lage, sich auf veränderliche Randbedingungen und auch auf Eingriffe des Menschen jederzeit einzustellen. Wir werden nicht überall vollautomatisierte Prozesse haben, stattdessen werden es veränderliche Prozesse sein, und hier ist die Möglichkeit des Menschen gefragt, direkt mit der Technik zu kommunizieren. Das heißt dass Technik den Menschen verstehen muss, wie auch der Mensch die Technik verstehen muss, und das möglichst auf eine intuitive Art und Weise.

In der Produktionswelt der Zukunft müssen Maschinen also in der Lage sein, sensorische Rückmeldungen eines Menschen zu verarbeiten - das kann bis hin zur Steuerung durch Gedanken gehen. Auf der anderen Seite müssen die Maschinen die Fähigkeit besitzen, ihren internen Zustand nutzerfreundlich zu visualisieren.

Durch mobile Endgeräte können Mitarbeiter individualisierte Informationen abrufen und werden so für wichtige Kerngrößen der Anlagen sensibilisiert. So können sie beispielsweise - wie oben am Beispiel ESIMA dargestellt - den Energieverbrauch kontinuierlich überwachen, bei Unregelmäßigkeiten sofort eingreifen und den Verbrauch somit optimieren.

Mit den steigenden Ansprüchen in der Informationstechnik muss auch das Knowhow der Mitarbeiter entsprechend wachsen. Eine Anpassung des Weiterbildungsangebotes ist die logische Konsequenz daraus. Technische Entwicklungsziele der Industrie 4.0 müssen zusammen mit der neuen Arbeitsorganisation und den neuen Qualifizierungsbedürfnissen abgestimmt sein. Exzellente Ressourcen für Forschung und Entwicklung sowie die Verfügbarkeit von Facharbeitern sind für die Zukunftsfähigkeit des Unternehmens notwendiger denn je. 
Industrie 4.0 ist ein interdisziplinäres und komplexes Projekt, das ganzheitlich aus unterschiedlichen Perspektiven betrachtet werden muss. Neben der reinen Technologie sind bei der Aus- und Weiterbildung weitere Aspekte einzubeziehen, zum Beispiel die Frage, wie die Kommunikation zwischen Mensch und Maschine gestaltet werden kann. In vielen Bereichen müssen Fachkräfte daher anders aus- und weitergebildet werden. Fabrikplaner zum Beispiel benötigen auch Kenntnisse in der Informations- und der Produktionstechnologie; Techniker brauchen viel praktische mechatronische Erfahrung, damit sie auf höchstem Niveau sehr schnell den Stillstand einer Anlage beheben können. Zudem kommt es darauf an, dass Ingenieure und Softwareentwickler eng zusammenarbeiten, denn hinter den intelligenten Maschinen steckt natürlich immer eine sehr gute Software. Daher muss der Softwareentwicklung im Maschinenbau mehr Aufmerksamkeit geschenkt werden.

Um diese Herausforderungen zu meistern, benötigen wir das Know-how der Ingenieure. Ihre Innovationskraft wird zum entscheidenden Wettbewerbsfaktor werden - in Zukunft werden die Unternehmen erfolgreich sein, die über genügend ausgebildete Fachkräfte sowie exzellente Ressourcen für Forschung und Entwicklung verfügen. Es wird daher immer wichtiger, dass technische Fachkräfte ihre Karrieren interdisziplinär aufbauen. Einsteiger haben im Bereich der Industrie 4.0 ungeahnte Möglichkeiten, Alleinstellungsmerkmale zu entwickeln, die sie für den Arbeitsmarkt sehr attraktiv machen werden.

Die Aufgaben- und Kompetenzprofile der Mitarbeiter werden sich in der Industrie 4.0 stark verändern; diese Prognose möchte ich wagen, auch wenn diese Veränderungen im Einzelnen noch nicht bekannt sein können, auch weil sie von den oben besprochenen Entscheidungen über Zukunftsszenarien abhängen. Das macht adäquate Qualifizierungsstrategien und - neben der schon genannten formalen Weiterbildung - eine lernförderliche Arbeitsorganisation notwendig, die arbeitsintegriertes Lernen ermöglicht.

Forschungs- und Entwicklungsbedarfe im Bereich der Methoden der Qualifikationsvorausschau wurden schon angesprochen. Weitere Forschungs- und Entwicklungsbedarfe beziehen sich auf neue Formen des Lernens, implizit in der Arbeit oder auch vermittelt durch neue digitale Lerntechnologien, die durchaus auch direkt mit der Produktionstechnik verschmolzen sein können. Auch die technischen Visionen, die in diesem Beitrag hier und da anklangen, sind noch keine ,Lösungen von der Stange', auch sie benötigen noch intensive Forschung und Entwicklung.

Neben Forschungs- und Entwicklungsprojekten werden für eine gute Gestaltung der Industrie 4.0 auch ,Netzwerke guter Praxis` erforderlich sein, um die Breite der Industrie einzubeziehen.

Ob Forschung und Entwicklung oder Erfahrungsaustausch über gute Praxis - Festo wird sich mit seinen Kompetenzen und mit vollem Engagement daran beteiligen, weil uns das Thema Industrie 4.0 in allen seinen Facetten - Technik, Mensch und Organisation sehr wichtig ist.

Open Access This chapter is distributed under the terms of the Creative Commons Attribution Noncommercial License, which permits any noncommercial use, distribution, and reproduction in any medium, provided the original author(s) and source are credited. 


\section{Literaturverzeichnis}

Bey, I., Luczak, H., Hinz, S., \& Quaas, W. (Hrsg.) (1995). Experte Mitarbeiter - Strategien und Methoden einer mitarbeiterorientierten Gestaltung und Einführung rechnerintegrierter Produktion. Köln: TÜV Rheinland.

Blum, U., \& Hartmann, E. A. (1988). Facharbeiterorientierte CNC-Steuerungs- und -Vernetzungskonzepte. Werkstatt und Betrieb, 121, 441-445.

Hartmann, E. A., \& Bovenschulte, M. (2013). Skills needs analysis for "Industry 4.0" based on roadmaps for smart systems. In International Labour Organization (ILO) \& SKOLKOVO Moscow School of Management (Eds.) Using technology foresights for identifying future skills needs. Global workshop proceedings. Moscow: SKOLKOVO Moscow School of Management.

Promotorengruppe Kommunikation der Forschungsunion Wirtschaft - Wissenschaft (Hrsg.) (2013). Deutschlands Zukunft als Produktionsstandort sichern - Umsetzungsempfehlungen für das Zukunftsprojekt Industrie 4.0. Abschlussbericht des Arbeitskreises Industrie 4.0. Online: http://www.bmbf.de/pubRD/Umsetzungsempfehlungen_Industrie4_0.pdf

Sell, R., \& Fuchs-Frohnhofen, P. (1993). Gestaltung von Arbeit und Technik durch Beteiligungsqualifizierung. Opladen: Westdeutscher Verlag. 